\title{
NOTE ON GROUP POSTULATES*
}

\section{BY HERBERT BOGGS AND G. Y. RAINICH}

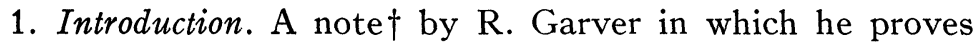
(following Huntington) that the existence of the product may be deduced from the existence of the right and left quotients and the associative law of multiplication suggests that it might be interesting to consider division (or the two divisions in the non-commutative case) as the fundamental operation, and to formulate a system of postulates in terms of that operation so that multiplication does not appear in the postulates at all. This naturally leads to a translation of the associative law of multiplication into a form which involves divisions only. That is what we do in the following paragraphs. A system of group postulates in terms of division has been given previously by Morgan Ward, $\ddagger$ but Ward introduces only one division (let us say the right division) and as a result of that his system seems to lack symmetry and does not contain a direct equivalent of the associative law of multiplication.

2. Proposed Postulates. The postulates are as follows.

I. To every two elements $a$ and $b$ of $G$ there corresponds $a$ unique element $c=a / b$, called the right quotient of a divided by $b$.

II. To every two elements $a$ and $b$ of $G$ there corresponds $a$ unique element $d=b \backslash a$, called the left quotient of a divided by $b$.

III. $a / b=c$ means the same as $c \backslash a=b$.

IV. (Associative law) $(a \backslash b) / c=a \backslash(b / c)$.

The independence of this set (or rather of a set obtained from these postulates by slight modification to insure the possibility of considering each independently from the others) is proved very easily by exhibiting, as does Ward, realizations in which all but one of the postulates is satisfied. For instance, if we interpret $G$ as the system of rational integers, $a / b$ as the sum $a+b$, $c \backslash a$ as the difference $a-c$, Postulates I, II, IV are satisfied but Postulate III is not.

\footnotetext{
* Presented to the Society, April 10, 1936.

$\dagger$ This Bulletin, vol. 40 (1934), pp. 698-701.

$\ddagger$ Transactions of this Society, vol. 32 (1930), pp. 520-526.
} 
Our purpose is now to prove, given two elements $a$ and $b$ of $G$, the existence and uniqueness of an element $x$ of $G$ which satisfies the relations

$$
a \backslash x=b \quad \text { and } \quad x / b=a,
$$

and which we shall call the product $a b$. This will be done with the aid of three lemmas.

3. Three Lemmas. We shall prove first the following lemma.

Lemma 1. For any two elements $a$ and $b$

$$
a \backslash a=b \backslash b, \quad a / a=b / b .
$$

Proof. Introduce (Postulates I and II)

$$
c=a / b, \quad e=a \backslash a .
$$

By Postulate III,

$$
b=c \backslash a, \quad a=a / e .
$$

By Postulate IV, we have

$$
(c \backslash a) / e=c \backslash(a / e),
$$

and with the aid of (2) and (3) this gives $b / e=b$, which, by Postulate III, gives $e=b \backslash b$. This proves the first part of the lemma. The second part is proved in an analogous way.

LEMMA 2. For any element $a$ in $G$

$$
a / a=a \backslash a .
$$

Proof. According to Lemma 1 it is sufficient to prove this for some one element of $G$. Starting with an arbitrary $a$ we introduce by Postulate I

$$
e=a / a .
$$

By the second part of Lemma $1, a / a=e / e$, which with (4) gives $e / e=e$. By Postulate III, this means $e=e \backslash e$, but according to the first part of Lemma $1, e \backslash e=a \backslash a$. Therefore $a / a=a \backslash a$ and the lemma is proved.

Definition. The element $a / a=a \backslash a$ which is independent of $a$ and whose existence is proved by the foregoing lemmas is called the unit element and is denoted by $e$, so that we have 


$$
a / a=a \backslash a=e,
$$

and according to Postulate III,

$$
a=a / e=e \backslash a .
$$

CoRollary 1. If $a / b=e$ or if $b \backslash a=e$, then $a=b$.

Proof. From Postulate III, $b=e \backslash a$ or $b=a / e$. In each case, from (6), $a=b$.

Lemma 3. (Second form of associative law) For any three elements $a, b$, and $c$,

$$
(a / b) \backslash c=b /(c \backslash a) .
$$

Proof. Introduce (Postulates I and II) the elements

$$
p=a / b, \quad q=c \backslash a .
$$

By Postulate III these relations may be written

$$
p \backslash a=b, \quad a / q=c .
$$

By Postulate IV, we have $(p \backslash a) / q=p \backslash(a / q)$, which with (8) and (9) gives (7).

4. Multiplication. To establish the existence of the product consider now the expression $[(e / a) \backslash b] / b$. By Postulate IV, it is equal to $(e / a) \backslash(b / b)$. By (5), it may be written as $(e / a) \backslash e$, or, applying Lemma $3, a /(e \backslash e)$, which, by (5), becomes $a / e$, and according to (6) is equal to $a$. The result, which may be written

$$
[(e / a) \backslash b] / b=a,
$$

establishes the existence of the product, as defined above, for any two elements $a$ and $b$; this product is given explicitly by

$$
a b=(e / a) \backslash b \text {. }
$$

We next prove the uniqueness of multiplication; in other words, we want to prove that if $x / a=b$ and $y / a=b$, we must have $x=y$. According to (5),

$$
(x / a) \backslash(y / a)=e .
$$

Using the associative law (Postulate IV), we may write this as

$$
[(x / a) \backslash y] / a=e .
$$


According to Corollary 1, $(x / a) \backslash y=a$. Using Lemma 3 (second form of associative law), we have $a /(y \backslash x)=a$, or by Postulate III, $y \backslash x=a \backslash a$. By definition of $e$ and Corollary 1, this gives $y=x$.

5. Conclusion. We use four postulates, whereas Huntington and Garver have shown that three are sufficient. This may seem to be a disadvantage for those who seek to reduce the number of postulates to a minimum. This is not the point of view we take; four postulates may be always combined into one by inserting three "ands" in the proper places. It may be thought that this would be an artificial combination, but it would be very difficult to define "artificial." As opposed to this view we hold that there may be an advantage in splitting up postulates into independent postulates; we think this amounts to a keener analysis of the situation. If multiplication is introduced into the postulates (although the existence of a product is not postulated) and the two divisions are defined in terms of it, this is equivalent to assuming our Postulate III without making it explicit. By discarding multiplication we make it possible to consider situations in which two divisions exist but are not connected by Postulate III, as in the above example proving the independence of Postulate III.

The fact that there seems to be an advantage in considering division as the fundamental operation is not surprising if one thinks of Euclid's (or Eudoxus') theory of proportions, in which the ratio of two segments is fundamental, or of the geometrical calculus in which vectors are introduced as the differences of points, and addition is of secondary importance.

The University of Michigan 